\title{
$\beta$-TRANSFORMATION, INVARIANT MEASURE AND UNIFORM DISTRIBUTION
}

\section{GAVIN BROWN and QINGHE YIN}

(Received 18 December 1997; revised 8 April 1999)

Communicated by A. H. Dooley

\begin{abstract}
Let $T_{\beta}$ be the $\beta$-transformation on $[0,1)$. When $\beta$ is an integer $T_{\beta}$ is ergodic with respect to Lebesgue measure and almost all orbits $\left\{T_{\beta}^{n} x\right\}$ are uniformly distributed. Here we consider the non-integer case, determine when $T_{\alpha}, T_{\beta}$ have the same invariant measure and when (appropriately normalised) orbits are uniformly distributed.
\end{abstract}

1991 Mathematics subject classification (Amer. Math. Soc.): primary 28D05, $11 \mathrm{~K} 55,11 \mathrm{~K} 06$.

Keywords and phrases: $\beta$-transformation, invariant measure, uniform distribution..

\section{Introduction and results}

Let $\beta>1$ be a real number. The $\beta$-transformation is the map $T_{\beta}:[0,1) \mapsto[0,1)$ given by

$$
T_{\beta} x=\beta x-[\beta x], \text { for all } x \in[0,1)
$$

where $[t]$ is the largest integer which is not greater than $t$. Ergodic properties of $\beta$-transformations are studied by many authors (see [1,3-5,8]). For each $\beta>1$, $T_{\beta}$ possesses a probability invariant measure, $\mu_{\beta}$, which is equivalent to Lebesgue measure, and $T_{\beta}$ is ergodic with respect to $\nu_{\beta}$. We ask when is $\mu_{\alpha}=\mu_{\beta}$ ? The related map $S_{\beta}$ on $[0,1)$, defined by

$$
S_{\beta} x= \begin{cases}T_{\beta} x & \text { if } x<[\beta] / \beta, \\ (x-[\beta]) /(\beta-[\beta]) & \text { if } x \geq[\beta] / \beta,\end{cases}
$$

(C) 1999 Australian Mathematical Society 0263-6115/99 $\$$ A2.00+0.00 
preserves Lebesgue measure and is ergodic (see [2, pp. 168-172]). For $x \in[0,1$ ), we define a sequence

$$
y_{n}(\beta)= \begin{cases}T_{\beta}^{n} x & \text { if } T_{\beta}^{n-1} x<[\beta] / \beta, \\ T_{\beta}^{n} x /(\beta-[\beta]) & \text { if } T_{\beta}^{n-1} x \geq[\beta] / \beta,\end{cases}
$$

and ask when is $\left\{y_{n}(\beta)\right\}$ uniformly distributed for almost all $x \in[0,1)$ ?

In the case where $\alpha, \beta$ are integers it is well-known that $\nu_{\alpha}=v_{\beta}$ is Lebesgue measure and that $\left\{y_{n}(\beta)\right\}$ is uniformly distributed for almost all $x$, so the interested cases are non-integer cases. Let

$$
\mathscr{A}=\left\{\beta: \beta>1 \text { satisfies } x^{2}-k x-l=0, k, l \in \mathbb{Z}, k \geq l \geq 1\right\} .
$$

Our results can be stated as follows.

THEOREM 1. Suppose that $\beta>1$ is not an integer. If $\beta \in \mathscr{A}$ then we have $\mu_{\beta}=\mu_{\beta+1}$. For any other $\alpha \neq \beta$ we have $\mu_{\alpha} \neq \mu_{\beta}$.

THEOREM 2. Suppose that $\beta>1$ is not an integer. If $\beta \in \mathscr{A}$ then for almost all $x,\left\{y_{n}\right\}$ is uniformly distributed. If $\beta \notin \mathscr{A}$ then for almost all $x,\left\{y_{n}\right\}$ is not uniformly distributed.

For $x \in[0,1)$, we define $x_{n}(\beta)=T_{\beta}^{n} x$. By the ergodicity of $T_{\beta}$, for almost all $x$, the sequence $\left\{x_{n}\right\}$ is $\mu_{\beta}$-distributed. When $\beta$ is an integer, since $\mu_{\beta}$ is the Lebesgue measure restricted to $[0,1)$, then $\left\{x_{n}(\beta)\right\}$ is uniformly distributed for almost all $x \in[0,1)$. We may rewrite the definition of $\left\{y_{n}(\beta)\right\}$ as

$$
y_{n}(\beta)= \begin{cases}x_{n} & \text { if } x_{n-1}<[\beta] / \beta, \\ x_{n} /(\beta-[\beta]) & \text { if } x_{n-1} \geq[\beta] / \beta .\end{cases}
$$

Clearly, if $\beta$ is an integer then $\left\{x_{n}\right\}$ and $\left\{y_{n}\right\}$ coincide. We may define $z_{n}(\beta)=S_{\beta}^{n} x$. Since $S_{\beta}$ preserves Lebesgue measure, we see that $\left\{z_{n}(\beta)\right\}$ is uniformly distributed for almost all $x$. Comparing the definitions of $\left\{y_{n}(\beta)\right\}$ and $S$, it may seem plausible that, for any $\beta>1,\left\{y_{n}(\beta)\right\}$ should be uniformly distributed for almost all $x$.

It is also interesting to compare Theorem 2 with the results of [6]. Schweiger in 1972 studied sequences similar to our $\left\{y_{n}(\beta)\right\}$ for some special Oppenheim series $[6,7]$. The Oppenheim series is defined as follows: Let $a_{n}$ be a decreasing sequence with $a_{1}=1$ and $\lim _{n \rightarrow \infty} a_{n}=0$. Let $b_{n} \geq 1$. The map $T:[0,1) \mapsto[0,1)$ is piecewise defined as

$$
T x=\frac{x-a_{n+1}}{b_{n}\left(a_{n}-a_{n+1}\right)}, \quad x \in\left[a_{n+1}, a_{n}\right) .
$$


We define $T 0=0$, if necessary.

For $x \in[0,1)$, let $u_{n}=T^{n} x$ and $v_{n}=b_{k_{n}} u_{n}$, if $u_{n-1} \in\left[a_{k_{n}+1}, a_{k_{n}}\right)$. Schweiger [6] showed that in cases

1. $a_{n}=1 / n, b_{n}=1$ (Lüroth's series);

2. $a_{n}=1 / n, b_{n}=n$ (Engel's series) and

3. $a_{n}=1 / n, b_{n}=n(n+1)$ (Sylvester's series)

$\left\{v_{n}\right\}$ is uniformly distributed for almost all $x \in[0,1)$.

If, for non-integral $\beta>1$, we let $a_{1}=1, a_{n}=([\beta]+2-n) / \beta, n=2, \ldots,[\beta]+2$ and $a_{n}=0$ for $n>[\beta]+2$, and let $b_{1}=1 /(\beta-[\beta])$ and $b_{n}=1$ for $n>1$, then the map $T$ is just the $\beta$-transformation $T_{\beta}$, and $\left\{v_{n}\right\}$ is just $\left\{y_{n}(\beta)\right\}$. Again, this may suggest the possibility of uniform distribution of $\left\{y_{n}(\beta)\right\}$.

Before giving the proofs let us develop some background concerning $\beta$-expansions. For $x \in[0,1)$ we have

$$
x=\frac{c_{1}}{\beta}+\frac{c_{2}}{\beta^{2}}+\cdots
$$

where $c_{n}=\left[\beta T_{\beta}^{n-1} x\right]$. Equation (1.1) is called the $\beta$-expansion of $x$. Suppose that the $\beta$-expansion of $\beta-[\beta]$ is

$$
\beta-[\beta]=\frac{\varepsilon_{2}}{\beta}+\frac{\varepsilon_{3}}{\beta^{2}}+\cdots .
$$

Then we have

$$
1=\frac{\varepsilon_{1}}{\beta}+\frac{\varepsilon_{2}}{\beta^{2}}+\frac{\varepsilon_{3}}{\beta^{3}}+\cdots
$$

where $\varepsilon_{1}=[\beta]$ and (1.2) is called the $\beta$-expansion of 1 . Notice that to say $\beta \in \mathscr{A}$ is equivalent to say that the $\beta$ expansion of 1 is

$$
1=\frac{k}{\beta}+\frac{l}{\beta^{2}}
$$

We also denote $T_{\beta}^{0} 1=1, T_{\beta} 1=\beta-[\beta]$ and $T_{\beta}^{n} 1=T_{\beta}\left(T_{\beta}^{n-1} 1\right)$ for $n \geq 2$. $\beta$ expansions have the following properties:

(P) Let (1.2) be the $\beta$-expansion of 1. For any $x \in[0,1)$ with the $\beta$-expansion given by (1.1) and any $n \geq 1$ we have

$$
\left(c_{n}, c_{n+1}, c_{n+2}, \ldots\right)<\left(\varepsilon_{1}, \varepsilon_{2}, \varepsilon_{3}, \ldots\right)
$$

where ' $<$ ' is according to the lexicographical order. 
By (P) we get that for any $n \geq 2$

$$
\left(\varepsilon_{n}, \varepsilon_{n+1}, \varepsilon_{n+2}, \ldots\right)<\left(\varepsilon_{1}, \varepsilon_{2}, \varepsilon_{3}, \ldots\right) .
$$

The absolutely continuous invariant measure for $T_{\beta}, \mu_{\beta}$, can be defined as follows (see [4]). Let

$$
h_{\beta}(x)=\sum_{x<T_{\beta}^{n} 1, n \geq 0} \frac{1}{\beta^{n}} .
$$

Then for any Borel subset $E$ of $[0,1)$,

$$
\mu_{\beta}(E)=\frac{1}{c_{\beta}} \int_{B} h_{\beta}(x) d x,
$$

where $c_{\beta}=\int_{0}^{1} h_{\beta}(x) d x$ is the normalizing constant.

Theorem 1 will be proved in Section 2 and Theorem 2 in Section 3.

\section{Proof of Theorem 1}

PROOF. Assume that $\beta>1$ is not an integer and that

$$
1=\frac{k}{\beta}+\frac{l}{\beta^{2}}
$$

where $k \geq l>0$. Then

$$
T_{\beta}^{0} 1=1, \quad T_{\beta} 1=\frac{l}{\beta}, \quad \text { and } \quad T_{\beta}^{n} 1=0 \quad \text { for } n \geq 2 .
$$

Hence

$$
h_{\beta}(x)= \begin{cases}1+\frac{1}{\beta} & \text { if } 0 \leq x<\frac{l}{\beta} \\ 1 & \text { if } \frac{l}{\beta} \leq x<1\end{cases}
$$

For $\beta+1$ we have

$$
T_{\beta+1}^{0} 1=0, \quad T_{\beta+1} 1=\beta+1-[\beta+1]=\beta-[\beta]=\frac{l}{\beta}
$$

and

$$
T_{\beta+1}^{2}=(\beta+1) \frac{l}{\beta}-\left[(\beta+1) \frac{l}{\beta}\right]=\frac{l}{\beta} .
$$


Hence for any $n \geq 1$ we have

$$
T_{\beta+1}^{n} 1=\frac{l}{\beta}
$$

Therefore

$$
\begin{aligned}
h_{\beta+1}(x) & = \begin{cases}1+\frac{1}{\beta+1}+\frac{1}{(\beta+1)^{2}}+\cdots & \text { if } 0 \leq x<\frac{l}{\beta}, \\
1 & \text { if } \frac{l}{\beta} \leq x<1\end{cases} \\
& = \begin{cases}1+\frac{1}{\beta} & \text { if } 0 \leq x<\frac{l}{\beta}, \\
1 & \text { if } \frac{l}{\beta} \leq x<1\end{cases} \\
& =h_{\beta}(x) .
\end{aligned}
$$

This shows that $\mu_{\beta}=\mu_{\beta+1}$.

On the other hand, suppose that $\mu_{\alpha}=\mu_{\beta}$ for some $\alpha>1$. Then we must have

$$
\sum_{x<T_{\alpha}^{n} 1, n \geq 0} \frac{1}{\alpha^{n}}=h_{\alpha}(x)=h_{\beta}(x)= \begin{cases}1+\frac{1}{\beta} & \text { if } 0 \leq x<\frac{l}{\beta}, \\ 1 & \text { if } \frac{l}{\beta} \leq x<1 .\end{cases}
$$

Therefore $\alpha$ must satisfy one of the following two cases:

(a) $T_{\alpha} 1=l / \beta$ and $T_{\alpha}^{n} 1=0$ for $n \geq 2$, or

(b) $T_{\alpha}^{n} 1=l / \beta$ for all $n \geq 1$.

In case (a) we have

$$
h_{\alpha}(x)= \begin{cases}1+\frac{1}{\alpha} & \text { if } 0 \leq x<\frac{l}{\beta} \\ 1 & \text { if } \frac{l}{\beta} \leq x<1\end{cases}
$$

which gives $\alpha=\beta$.

In cases (b) we obtain

$$
\begin{aligned}
h_{\alpha}(x) & = \begin{cases}1+\frac{1}{\alpha}+\frac{1}{\alpha^{2}}+\cdots & \text { if } 0 \leq x<\frac{l}{\beta} \\
1 & \text { if } \frac{l}{\beta} \leq x<1\end{cases} \\
& = \begin{cases}1+\frac{1}{\alpha-1} & \text { if } 0 \leq x<\frac{l}{\beta} \\
1 & \text { if } \frac{l}{\beta} \leq x<1\end{cases}
\end{aligned}
$$


which yields $\alpha=\beta+1$.

Now we assume that $\beta \notin \mathscr{A}$. First we assume that the $\beta$-expansion of 1 is

$$
1=\frac{\varepsilon_{1}}{\beta}+\frac{\varepsilon_{2}}{\beta^{2}}+\cdots+\frac{\varepsilon_{m}}{\beta^{m}},
$$

where $m \geq 3$ and $\varepsilon_{m}>0$. Suppose that there exists $\alpha \neq \beta$ such that $\mu_{\alpha}=\mu_{\beta}$. If the $\alpha$-expansion of 1 is

$$
1=\frac{e_{1}}{\alpha}+\frac{e_{2}}{\alpha^{2}}+\cdots+\frac{e_{n}}{\alpha^{n}}, e_{n}>0
$$

then we would have $\alpha=\beta$. In fact if (2.1) holds, then we have $T_{\alpha}^{i} 1 \neq T_{\alpha}^{j} 1$ for $0 \leq i<j \leq n$. Since $\mu_{\alpha}=\mu_{\beta}$, by (2.1) we see that the density function of $\mu_{\alpha}$ is a step function with $m$ pieces. Hence we must have $n=m$. Thus

$$
1+\frac{1}{\beta}+\cdots+\frac{1}{\beta^{m-1}}=h_{\beta}(0)=h_{\alpha}(0)=1+\frac{1}{\alpha}+\cdots+\frac{1}{\alpha^{m-1}}
$$

which gives $\alpha=\beta$. Thereafter we may assume that the $\alpha$-expansion of 1 is

$$
1=\frac{e_{1}}{\alpha}+\frac{e_{2}}{\alpha^{2}}+\cdots
$$

where there are infinitely many $e_{n}>0$. Then we get

$$
1+\frac{1}{\beta}+\cdots+\frac{1}{\beta^{m-1}}=h_{\beta}(0)=h_{\alpha}(0)=1+\frac{1}{\alpha}+\frac{1}{\alpha^{2}}+\cdots
$$

which gives

$$
\alpha=\frac{\beta^{m}-1}{\beta^{m-1}-1}>\beta .
$$

We have $T_{\beta}^{m-1} 1=\varepsilon_{m} / \beta$. Then there must exist $i \leq m$ such that

$$
\frac{\varepsilon_{m}}{\beta}=T_{\beta}^{m-1} 1=T_{\alpha}^{i-1} 1=\frac{e_{i}}{\alpha}+\frac{e_{i+1}}{\alpha^{2}}+\cdots
$$

Then the right hand side of (2.4) is the $\alpha$-expansion of $\varepsilon_{m} / \beta$. Since $\alpha>\beta$ we get $e_{i} \geq \varepsilon_{m}$. By (2.3) and (2.4) we obtain

$$
\begin{aligned}
\frac{\varepsilon_{m}}{\beta}+ & \frac{\varepsilon_{m}}{\beta^{2}}+\cdots+\frac{\varepsilon_{m}}{\beta^{m-1}} \\
= & \left(\frac{e_{i}}{\alpha}+\frac{e_{i+1}}{\alpha^{2}}+\cdots\right)+\frac{1}{\beta}\left(\frac{e_{i}}{\alpha}+\frac{e_{i+1}}{\alpha^{2}}+\cdots\right)+\cdots+\frac{1}{\beta^{m-1}}\left(\frac{e_{i}}{\alpha}+\frac{e_{i+1}}{\alpha^{2}}+\cdots\right) \\
> & \frac{e_{i}}{\alpha}+\frac{e_{i}+e_{i+1}}{\alpha^{2}}+\frac{e_{i}+e_{i+1}+e_{i+2}}{\alpha^{3}}+\cdots+\frac{e_{i}+e_{i+1}+\cdots+e_{i+m-2}}{\alpha^{m-1}} \\
& +\frac{e_{m+1}+e_{m+2}+\cdots+e_{i+m-1}}{\alpha^{m}}+\frac{e_{i+2}+e_{i+3}+\cdots+e_{i+m}}{\alpha^{m+1}}+\cdots
\end{aligned}
$$

We use $A$ to denote the last expression. There are two possibilities: 
424

Gavin Brown and Qinghe Yin

[7]

(a) There exists $j$ with $i+1 \leq j \leq i+m-2$ such that $e_{j}>0$, or

(b) $e_{i+1}=e_{i+2}=\cdots=e_{i+m-2}=0$.

In case (a) we have

$$
\begin{aligned}
A & \geq \frac{\varepsilon_{m}}{\alpha}+\frac{\varepsilon_{m}}{\alpha^{2}}+\cdots+\frac{\varepsilon_{m}}{\alpha^{m-1}}+\frac{e_{j}}{\alpha^{j-i+1}}+\frac{e_{j}}{\alpha^{j-i+2}}+\cdots+\frac{e_{j}}{\alpha^{j+i+m-1}} \\
& \geq \frac{\varepsilon_{m}}{\alpha-1}\left(1-\frac{1}{\alpha^{m-1}}\right)+\frac{1}{1-\alpha} \cdot \frac{1}{\alpha^{j-i}}\left(1-\frac{1}{\alpha^{m-1}}\right) \\
& \geq \frac{\varepsilon_{m}}{\alpha-1}\left(1-\frac{1}{\alpha^{m-1}}\right)+\frac{1}{1-\alpha} \cdot \frac{1}{\alpha^{m-2}}\left(1-\frac{1}{\alpha^{m-1}}\right) .
\end{aligned}
$$

On the other hand, we have

$$
\frac{\varepsilon_{m}}{\beta}+\frac{\varepsilon_{m}}{\beta^{2}}+\cdots+\frac{\varepsilon_{m}}{\beta^{m-1}}=\frac{\varepsilon_{m}}{\alpha}+\frac{\varepsilon_{m}}{\alpha^{2}}+\cdots=\frac{\varepsilon_{m}}{\alpha-1} .
$$

If we can show that

$$
\frac{1}{\alpha^{m-2}}\left(1-\frac{1}{\alpha^{m-1}}\right) \geq \frac{\varepsilon_{m}}{\alpha^{m-1}}
$$

then we get a contradiction:

$$
\frac{\varepsilon_{m}}{\beta}+\frac{\varepsilon_{m}}{\beta^{2}}+\cdots+\frac{1}{\beta^{m-1}}>A \geq B .
$$

Inequality (2.5) is equivalent to

$$
\alpha^{m-1}-1 \geq \varepsilon_{m} \alpha^{m-2}
$$

Since $\varepsilon_{m} \leq \varepsilon_{1}=[\beta] \leq[\alpha]$, it is enough to show

$$
\alpha^{m-1}-1 \geq[\alpha] \alpha^{m-2}
$$

which is equivalent to

$$
1 \geq \frac{[\alpha]}{\alpha}+\frac{1}{\alpha^{m-1}}
$$

Thus if (2.6) holds we have $\mu_{\alpha} \neq \mu_{\beta}$.

If (2.6) does not hold, since there are only $m-1$ choices for $T_{\alpha}^{i} 1, i \geq 1$, then we get

$$
1=\frac{[\alpha]}{\alpha}+\frac{l}{\alpha^{m}}+\frac{l}{\alpha^{2 m-1}}+\cdots,
$$

https://doi.org/10.1017/S1446788700036703 Published online by Cambridge University Press 
for some $1 \leq l<[\alpha]$. This is included in case (b).

In case (b) we have

$$
1=\frac{e_{1}}{\alpha}+\frac{e_{2}}{\alpha^{2}}+\cdots+\frac{e_{i}}{\alpha^{i}}+\frac{e_{i+m-1}}{\alpha^{i+m-1}}+\cdots .
$$

Since there are only $m-1$ possibilities for $T_{\alpha}^{j} 1, j \geq 1$, we deduce that

$$
1=\frac{e_{1}}{\alpha}+\frac{e_{i}}{\alpha^{i}}+\frac{e_{i}}{\alpha^{i+m-1}}+\frac{e_{i}}{\alpha^{i+2 m-2}}+\cdots \text {. }
$$

Then

$$
\begin{aligned}
T_{\alpha}^{i} 1 & =\frac{e_{i}}{\alpha^{m-1}}+\frac{e_{i}}{\alpha^{2 m-2}}+\cdots \\
& =\frac{e_{i}}{\alpha^{m-1}-1}<\frac{1}{\beta^{m-2}}
\end{aligned}
$$

Since $h_{\alpha}(x)=h_{\beta}(x)$ for each $1 \leq l \leq m-1$ there would exist $1 \leq k \leq m-1$ such that $T_{\beta}^{l} 1=T_{\alpha}^{k} 1$. Then by (2.7) we get

$$
1=\frac{\varepsilon_{1}}{\beta}+\frac{\varepsilon_{m}}{\beta^{m}} .
$$

In fact if (2.8) is not true then we have $T_{\beta}^{j} 1 \geq 1 / \beta^{m-2}$ for any $1 \leq j \leq m-1$. Now we have

$$
\frac{\varepsilon_{m}}{\beta}=T_{\beta}^{m-1} 1=T_{\alpha}^{i-1} 1
$$

and

$$
\begin{aligned}
\frac{\varepsilon_{m}}{\beta^{2}} & =T_{\beta}^{m-2} 1= \begin{cases}T_{\alpha}^{i-2} 1 & i \geq 3, \\
T_{\alpha}^{m-1} 1 & i=2\end{cases} \\
& =\frac{1}{\alpha} T_{\alpha}^{i-1} 1=\frac{1}{\alpha} \cdot \frac{\varepsilon_{m}}{\beta}
\end{aligned}
$$

which gives $\alpha=\beta$, a contradiction.

Now we consider those $\beta$ for which the $\beta$-expansion of 1 has infinitely many nonzero terms. By the above discussion, if $\mu_{\beta}=\mu_{\alpha}$ for some $\alpha$ then the $\alpha$ expansion of 1 must have infinitely many non-zero terms. Since $\mu_{\beta}=\mu_{\alpha}$ we have

$$
h_{\beta}(x)=c \cdot h_{\alpha}(x)
$$

for some constant $c$. Notice that we have

$$
\lim _{x \rightarrow 1} h_{\beta}(x)=1=\lim _{x \rightarrow 1} h_{\alpha}(x) .
$$


Then $c=1$. We also have

$$
\lim _{x \rightarrow 0} h_{\beta}(x)=1+\frac{1}{\beta}+\frac{1}{\beta^{2}}+\cdots=\frac{\beta}{\beta-1}
$$

and

$$
\lim _{x \rightarrow 0} h_{\alpha}(x)=1+\frac{1}{\alpha}+\frac{1}{\alpha^{2}}+\cdots=\frac{\alpha}{\alpha-1} .
$$

Therefore, we have $\alpha=\beta$ and the proof is complete.

\section{Proof of Theorem 2}

Proof. Let $\beta>1$ be a non-integer. Given a Borel set $E$, by the ergodicity of $T_{\beta}$, for almost all $x$ we have

$$
\lim _{N \rightarrow \infty} \frac{\left\{n: n \geq N, x_{n}(\beta) \in E\right\}}{N}=\mu_{\beta}(E) .
$$

For $0<\alpha \leq 1$, by definition, $y_{n}(\beta)<\alpha$ if and only if

$$
x_{n-1}(\beta) \in \bigcup_{i=0}^{[\beta]-1}\left[\frac{i}{\beta}, \frac{i+\alpha}{\beta}\right) \cup\left[\frac{[\beta]}{\beta}, \frac{[\beta]+(\beta-[\beta]) \alpha}{\beta}\right) .
$$

For convenience, we use $E_{\alpha}$ to denote the right hand side of (3.2). Now for almost all $x$ we have

$$
\begin{aligned}
& \lim _{N \rightarrow \infty} \frac{\left\{n: n \leq N, y_{n}(\beta) \leq \alpha\right\}}{N}=\mu_{\beta}\left(E_{\alpha}\right) \\
= & \sum_{i=0}^{[\beta]-1}\left(F\left(\frac{i+\alpha}{\beta}\right)-F\left(\frac{i}{\beta}\right)\right)+F\left(\frac{[\beta]+(\beta-[\beta]) \alpha}{\beta}\right)-F\left(\frac{[\beta]}{\beta}\right)
\end{aligned}
$$

where $F(t)=\mu_{\beta}(\{x<t\})$. Let $G(t)=\mu_{\beta}\left(E_{t}\right)$. Then

$$
G^{\prime}(t)=\frac{1}{\beta} \sum_{i=0}^{[\beta]-1} \rho\left(\frac{i+t}{\beta}\right)+\frac{\beta-[\beta]}{\beta} \rho\left(\frac{[\beta]+(\beta-[\beta]) t}{\beta}\right),
$$

where $\rho(x)=h_{\beta}(x) / c_{\beta}$ is the density function of $\mu_{\beta}$. In order that $\left\{y_{n}(\beta)\right\}$ be uniformly distributed, we need that $G^{\prime}(t) \equiv 1$. Noting that $\rho(t)$ is a decreasing step function and $G(t)$ is a distribution function, we obtain that $G^{\prime}(t) \equiv 1$ if and only if each term in the sum of the right hand side of (3.3) is a constant. 
If $\beta \in \mathscr{A}$ then the $\beta$-expansion of 1 is

$$
1=\frac{k}{\beta}+\frac{l}{\beta^{2}}
$$

and

$$
T_{\beta}^{0} 1=1, \quad T_{\beta}^{1} \beta=\frac{l}{\beta}, \quad \text { and } T_{\beta}^{n} 1=0, n \geq 2
$$

Hence

$$
h_{\beta}(x)= \begin{cases}1+1 / \beta & \text { if } x<l / \beta, \\ 1 & \text { otherwise. }\end{cases}
$$

In this case, each term of the right hand side of (3.3) is a constant. Therefore, $G^{\prime}(t) \equiv 1$ which implies that $\left\{y_{n}(\beta)\right\}$ is uniformly distributed for almost all $x$.

Now assume that $\beta \notin \mathscr{A}$. Then we have $T_{\beta} 1 \neq i / \beta$ for any $0 \leq i \leq[\beta]$. If $i / \beta<T 1<(i+1) / \beta$ where $0 \leq i \leq[\beta-1]$ then $h_{\beta}(i+t) / \beta$ is not a constant for $t \in[0,1)$. In fact if $t_{1}, t_{2}$ satisfies $\left(i+t_{1}\right) / \beta<T_{\beta} 1<\left(i+t_{2}\right) / \beta$ then

$$
h_{\beta}\left(\frac{i+t_{1}}{\beta}\right)-h_{\beta}\left(\frac{i+t_{2}}{\beta}\right) \geq \frac{1}{\beta} \text {. }
$$

If $[\beta] / \beta<T_{\beta}<1$ then for $t_{1}, t_{2} \in(0,1)$ with $\left([\beta]+(\beta-[\beta]) t_{1}\right) / \beta<T_{\beta} 1<$ $\left([\beta]+(\beta-[\beta]) t_{2}\right) / \beta$ we have

$$
h_{\beta}\left(\frac{[\beta]+(\beta-[\beta]) t_{1}}{\beta}\right)-h_{\beta}\left(\frac{[\beta]+(\beta-[\beta]) t_{2}}{\beta}\right) \geq \frac{1}{\beta} .
$$

In either case we have $G^{\prime}(t) \not \equiv 1$. This completes the proof.

\section{References}

[1] G. Brown and Q. Yin, ' $\beta$-transformation, natural extension and invariant measure', Ergodic Theory Dynamical Systems (to appear).

[2] I. P. Cornfeld, S. V. Fomin and Ya. G. Sinai, Ergodic theory (Springer, New York, 1982).

[3] S. Ito and Y. Takahashi, 'Markov subshifts and realization of $\beta$-expansions', J. Math. Soc. Japan 26 (1974), 33-55.

[4] W. Parry, 'On the $\beta$-expansion of real numbers', Acta Math. Hungar. 11 (1960), 401-416.

[5] A. Rényi, 'Representations of real numbers and their ergodic properties', Acta Math. Hungar. 8 (1957), 477-493.

[6] F. Schweiger, 'Metrische sätze über Oppenheimentwicklungen', J. Reine Angew. Math. 254 (1972), 152-159. 
[7] - Ergodic theory of fibred systems and metric number theory (Oxford University Press, Oxford, 1995).

[8] Y. Takahashi, 'Isomorphisms of $\beta$-automorphisms to Markov automorphisms', Osaka J. Math. 10 (1973), 157-184.

Vice-Chancellor

The University of Sydney

NSW 2006

Australia
School of Mathematics and Statistics The University of Sydney NSW 2006

Australia 